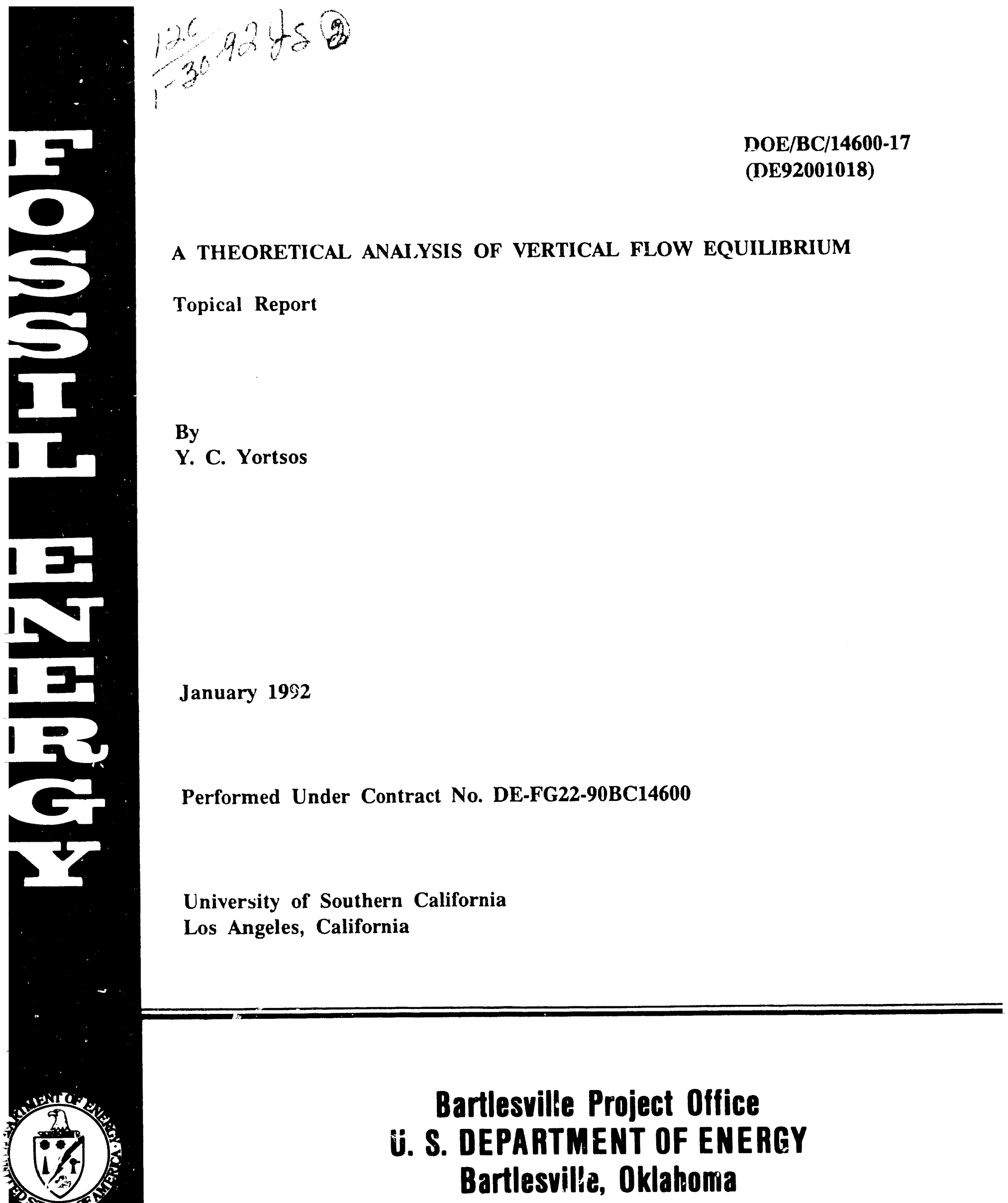




\section{DISCLAIMER}

This report was prepared as an account of work sponsored ty an agency of the United States Government. Neither the United States Government nor any agency thereof, nor any of their employees, makes any warranty, express or implied, or assumes any legal liability or responsibility for the accuracy, compieteness, or usefulness of any information, apparatus, product. or process disclosed, or represents that its use would not intringe privately owned rights. Reference herein to any specific commercial product. process, or service by trade name. trademark, manufacturer, or otherwise does not necessarily constitute or imply its er.dorsement, recommendation, or favoring by the United States Government or any agency thereof. The views and opinions of authors expressed herein do not necessarily state or reflect those of the United States Government or any agency thereof.

This report has been reproduced directly from the best available copy.

Available to DOE and DOE contractors from the Office of Scientific and Technical Information, P.O. Box 62, Oak Ridge, TN 37831; pi: :as available from (615)576-8401, FTS 626-8401.

Available to the public from the National Technical Information Service, U.S. Department of Commerce, 5285 Port Royal Rd., Springfield, VA 22161. 


\title{
A THEORETICAL ANALYSIS OF VERTICAL FLOW EQUILIBRIUM
}

Topical Report

By

Y. C. Yortsos

January 1992
$\mathrm{DOE} / \mathrm{BC} / 14600--17$

DE92 001018

Work Performed Under Contract No. FG22-90BC14600

Prepared for

U.S. Department of Energy

Assistant Secretary for Fossil Energy

Thomas B. Reid, Project Manager

Bartlesville Project Office

P. O. Box 1398

Bartlesville, OK 74005

\author{
Prepared by \\ University of Southern California \\ Department of Chemical Engineering \\ Petroleum Engineering Program \\ Los Angeles, CA 90089-1211
}

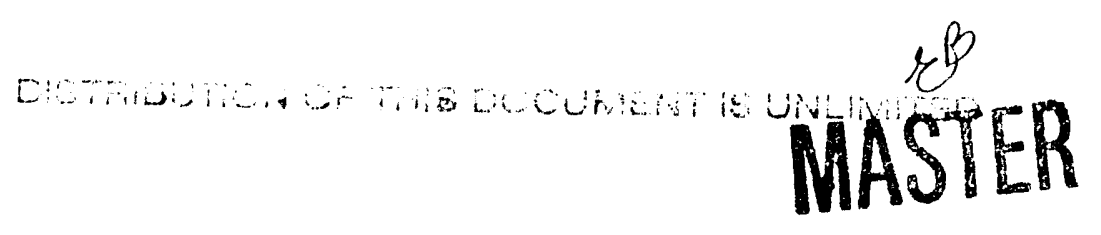




\section{Contents}

List of Figures

iv

List of Tables

1 Abstract

2 INTRODUCTION

3 ASYMPTOTIC ANALYSIS

4 LAYERED RESERVOIRS

5 ETFECTS OF CAPILLARITY AND GRAVITY

6 MISCIBLE DISPLACEMENT

7 TRACER DISPERSION

8 GRAVITY SEGREGATED FLOW

9 THE DUPUIT AND DIETZ APPROXIMATIONS

10 CAPILLARITY-GRAVITY SEGREGATION

11 SUMMARY $\mathbf{v}$

1

2

3

7

10

11

13

15

16

18

20 


\section{List of Figures}

1 Schematic of Heterogeneous Reservoir for VFE. . . . . . . . . . . . 23

2 Schematic of Viscous Fingering in VFE. . . . . . . . . . . . . . 23

3 Schematic of Gravity Segregated Displacement. . . . . . . . . . . . . . . . 25

4 Schematic of Layered Reservoir for VFE. . . . . . . . . . . . . . . . . . . . . 25

5 Schematic of Air-Water System for Dupuit Assumption. . . . . . . . . . . . 26 
List of Tables

1 


\section{A THEORETICAL ANALYSIS OF VERTICAL FLOW EQUILIBRIUM}

by

Y. C. Yortsos

\section{ABSTRACT}

The assumption of Vertical Flow Equilibrium (VFE) and of parallıl flow conditions, in general, is often applied to the modeling of flow and displacement in natural porous media. However, the methodology for the development of the various models is rather intuitive, and no rigorous method is currently available. In this paper, we develop an asymptotic theory using as parameter the variable $R_{L}=\frac{L}{H} \sqrt{\frac{k_{V}}{k_{H}}}$. It is rigorously shown that present models represent the leading order term of an asymptotic expansion with respect to $1 / R_{L}^{2}$. Although this was numerically suspected, it is the first time that it is theoretically proved. Based on the general formulation, a series of models are subsequently obtained. In the absence of strong gravity effects, they generalize previous works by Zapata and Lake (1981), Yokoyama and Lake (1981) and Lake and Hirasaki (1981), on immiscible and miscible displacements. In the limit of gravity-segregated flow, we prove conditions for the fluids to be segregated and derive the Dupuit and Dietz (1953) approximations. Finally, we also discuss effects of capillarity and transverse dispersion. 


\section{INTRODUCTION}

The description of displacement processes in oil reservoirs or water aquifers is often greatly simplified when the reservoir is narrow and long and the flow almost parallel. This is typically the case in many applications. Approximations under such conditions have been postulated by many researchers. In general, a Vertical Flow Equilibrium (VFE) is typically assumed (Fig. 1). Depending on the strength of gravity, the various approaches can be classified in two categories: One in which viscous forces and heterogeneity are predominant on the distribution of phases, and another in which the phases completely segregate due to gravity.

The first category is inten aed to capture primarily the effects of viscous forces and their interaction with heterogeneity (Fig. 2). It has been studied by several authors including Coats et al (1971), Yokoyama and Lake (1981), Zapata and Lake (1981), and more recently by Fande and Orr (1989) and Lake et al (1990). Since gravity is unimportant, the term vertical is meant to denote the direction along the narrow coordinate. In most of these studies a two-layer description is taken, using rather intuitive, although correct in retrospect, arguments. Extensive numerical simulation has verified the validity of the various approaches, particularly as it regards the dimensionless pa-

rameter $R_{L}=\frac{L}{H} \sqrt{\frac{k_{V}}{k_{H}}}$, which must take large enough values for the VFE to be applicable. Along the same lines must be considered the work by Lake and Hirasaki (1981) on tracer dispersion in stratified systems, as well various phenomenological viscous fingering models, such as Koval (1963), Todd and Longstaff (1972) and Fayers (1984). The latter models have presently only an empirical basis, although the numerical evidence is in many cases supportive of their applicability.

The second category emphasizes gravity in addition to viscous forces and it should be more applicable to homogeneous systems of higher permeability. Not surprising, the original contributions in this direction were made in connection with groundwater aquifers, where the so-called Dupuit assumption was introduced (see Bear, 1972). Viscous, two-phase flow was studied by Dietz (1953), and elaborated by Le Fur and Sourieau (1963), Beckers (1965) and others. A complete segregation of the immiscible phases is assumed, a sharp macroscopic interface separating the two regions (Fig. 3). Recently, Fayers and Muggeridge (1990) extended this approach to tilted reservoirs with dip.

While the two classes seen to derive from the same conditions, no effort has been taken to treat them in a uniform fashion. In fact, it is not entirety clear which are the relevant parameters that 
demarcate in the parameter space the two regimes and where do various approximations hold. At present, most of the available evidence is numerical. While under certain conditions, this may be sufficient, it would be nonetheless desirable if a rigorous derivation were available to clearly identify the various approximations and assumptions. This is particularly the case for layered systems, where presently available formalisms are awkward and difficult to extend to many layers.

The objective of this paper is to provide a unified approach based on a rigorous asymptotic expansion of the flow equations in long and narrow systems, where the VFE is expected to apply. First, the fundamental asymptotic analysis is developed for a model immiscible, two-phase displacement. In the absence of gravity or capillarity, an extension of the classical Buckley-Leverett equation, that also includes cross-flow terms, is obtained. Subsequently, this equation is applied to a layered systems of arbitrary number of layers. A hyperbolic non-linear system results that describes the interaction between the layers. Weak effects of capillarity and gravity are next introduced. The problem for miscible displacement is formulated in a subsequent section. Tracer dispersion in a layered system is analyzed leading to the results of Lake and Hirasaki (1981). Finally, we consider strong gravity and capillary effects that lead to segregated flow. The conditions for the latter are specified and we derive generalized expressions for the Dupuit and Dietz approximations. Flow segregation due to gravity-capillary equilibrium is also discussed.

\section{ASYMPTOTIC ANALYSIS}

Consider the constant rate immiscible displacement of "oil" by "water" in a long and narrow reservoir, of thickness $H$ and length $L$, where $\epsilon \equiv H / L \ll 1$. For simplicity, the reservoir has no dip, but it is anisotropic and stratified with different permeabilities in the "horizontal" and "vertical" directions

$$
K_{H}=k_{H} \kappa_{H}(X, Y) ; K_{V}=k_{V} \kappa_{V}(X, Y)
$$

Here $i_{2}>0,(i=H, V)$ are dimensionless such that $\int_{0}^{H} \kappa_{i} d Y=H$, when the $\mathrm{x}$-dependence is neglected. Otherwise, the latter constraint is not satisfied. We normalize "horizontal" and "vertical" scales, $X$ and $Y$, by $L$ and $H$, respectively: 


$$
x=\frac{X}{L} ; y=\frac{Y}{I I}
$$

and scale all velocities by the injection velocity $q$, time by $\frac{L}{q}$, and the fluid pressure by $\frac{L q \mu_{o}}{k_{H}}$. If $S$ denotes a "water" saturation, the dimensionless balances become

$$
\begin{array}{r}
\epsilon\left[\phi \frac{\partial S}{\partial t}+\frac{\partial u_{w}}{\partial x}\right]+\frac{\partial v_{w}}{\partial y}=0 \\
\epsilon \frac{\partial}{\partial x}\left(u_{w}+u_{o}\right)+\frac{\partial}{\partial y}\left(v_{w}+v_{o}\right)=0 \\
u_{i}=-\kappa_{H}(x, y) \lambda_{i} \frac{\partial p_{i}}{\partial x} ; i=o, w \\
\frac{\epsilon}{\delta} v_{i}=-\kappa_{V}(x, y) \lambda_{i}\left[\frac{\partial p_{i}}{\partial y}+\frac{\epsilon}{\delta} \frac{\rho_{i} k_{V} g}{q \mu_{o}}\right] ; i=o, w
\end{array}
$$

Here we defined $\delta \equiv \frac{k_{V}}{k_{H}}$, we have taken the $y$ coordinate to increase upwards, and we have used $u_{i}$ and $v_{i}$ to denote the "horizontal" and "vertical" components, respectively, of the dimensionless velocity of fluid $i$. In the absence of capillarity and gravity, $u_{i}$ and $v_{i}$ can be expressed in terms of the total velocities $u \equiv u_{w}+u_{o}$ and $v \equiv v_{w}+v_{o}$, with the use of the fractional flow function $f_{w}(S)$,

$$
u_{w}=u f_{w}(S) ; v_{w}=v f_{w}(S)
$$

We recall that $\lambda_{T}$ is the total mobility, $\lambda_{T} \equiv \lambda_{w}+\lambda_{o}$, where $\lambda_{w} \equiv \frac{\mu_{o}}{\mu_{w}} k_{r w}$ and $\lambda_{o} \equiv k_{r o}$, thus $f_{w} \equiv \lambda_{w} / \lambda_{T}$. We, then, obtain

$$
\begin{array}{r}
\epsilon\left[\phi \frac{\partial S}{\partial t}+\frac{\partial}{\partial x}\left(u f_{w}\right)\right]+\frac{\partial}{\partial y}\left(v f_{w}\right)=0 \\
\epsilon \frac{\partial u}{\partial x}+\frac{\partial v}{\partial y}=0 \\
u=-\kappa_{H}(x, y) \lambda_{T} \frac{\partial p}{\partial x} \\
\frac{\epsilon}{\delta} v=-\kappa_{V}(x, y) \lambda_{T} \frac{\partial p}{\partial y}
\end{array}
$$

Since $\epsilon \ll 1$ (long and narrow reservoirs), if follows that $u=O(1)$ and $v=O(\epsilon)$, hence we may define $v=\epsilon w$, where $w=O(1)$. Substitution into (5) then yiclds the following

$$
\phi \frac{\partial S}{\partial t}+u \frac{\partial f_{u}}{\partial x}+w \frac{\partial f_{w}}{\partial y}=0
$$




$$
\begin{array}{r}
\frac{\partial u}{\partial x}+\frac{\partial w}{\partial y}=0 \\
u=-\lambda_{T \kappa_{H}}(x, y) \frac{\partial p}{\partial x} \\
\frac{1}{R_{L}^{2}} w=-\lambda_{T} \kappa_{V}(x, y) \frac{\partial p}{\partial y}
\end{array}
$$

where we have defined $R_{L} \equiv \frac{\sqrt{\delta}}{\epsilon}=\frac{L}{H} \sqrt{\frac{k_{V}}{k_{H}}}$. In this formulation, it is now evident that the only dimensionless parameter is $R_{L}$, which is precisely the parameter used in justifying the use of VFE (Zapata and Lake, 1981). To obtain the latter approximation, we take the limit $R_{L}^{2} \gg 1$ and expand in a regular asymptotic expansion

$$
\begin{gathered}
u=u_{0}+\frac{1}{R_{L}^{2}} w_{1}+\cdots \\
w=w_{0}+\frac{1}{R_{L}^{2}} w_{1}+\cdots \\
p=p_{0}+\frac{1}{R_{L}^{2}} p_{1}+\cdots \\
S=S_{0}+\frac{1}{R_{L}^{2}} S_{1}+\cdots
\end{gathered}
$$

Substitution into (6) yields to leading order,

$$
\begin{aligned}
& \phi \frac{\partial S_{0}}{\partial t}+u_{0} \frac{\partial f_{w}}{\partial x}+w_{0} \frac{\partial f_{w}}{\text { partialy }}=0 \\
& \frac{\partial u_{0}}{\partial x}+\frac{\partial w_{0}}{\partial y}=0 \\
& u_{0}=-\lambda_{T} \kappa_{H}(x, y) \frac{\partial p_{0}}{\partial x} \\
& \frac{\partial p_{0}}{\partial y}=0
\end{aligned}
$$

Eqn (8) dictates that $p_{0}=p_{0}(x, t)$, i.e., the pressure depends on the $x$-coordinate only. This is the fundamental assumption of VFE or parallel flow, rigorously derived here in the limit $R_{L}^{2} \gg 1$. Numerical evidence suggests ti: t VFE is satisfied quite well when $R_{L} \geq 10$. This is consistent with our asymptotic expansion, where the next order enters at $O\left(\frac{1}{R_{L}^{2}}\right)$, hence the error made by retaining only the firct term is $1 \%$ or less. The rigorous identification of the parameter $R_{L}$ as the relevant variable for the validity of the VFE represents the first important result of this paper. 
We should point out that implicit in the derivation of $(8 d)$ was the assumption $\kappa_{V} \neq 0$. For a layered system, where the permeability $\kappa_{V}=\kappa v(y)$ may vanish somewhere, one simply needs to redefine the reservoir thickness and VFE applies without problem (Lake et al., 1990). The problem is somewhat more complicated, however, when the system is not layered. At least near the regions where $\kappa_{V}$ vanishes, the VFE does not apply. This may complicate the analysis, thus it will not be discussed further in this paper.

Unless otherwise noted, for convenience, we shall omit subscripts 0 and $H$. The next step is to eliminate $\frac{\partial p}{\partial x}$ by integrating (8c) along the $y$-coordinate, to obtain

$$
u=\frac{\lambda_{T}(S) \kappa(x, y)}{\int_{0}^{1} \lambda_{T}(S) \kappa(x, y) d y}
$$

where we have assumed a constant injection rate (although the extension to a variable rate is straightforward). Subsequently, we eliminate $w$ by integrating (8b)

$$
w=-\int_{0}^{y} \frac{\partial u}{\partial x} d y
$$

where a no-flow boundary condition was used. Final substitution into (8a) yields the result

$$
\phi \frac{\partial S}{\partial t}+\frac{\lambda_{T} \kappa}{\int_{0}^{1} \lambda_{T} \kappa d y} \frac{\partial f_{w}}{\partial x}-\frac{\partial}{\partial x}\left[\frac{\int_{0}^{y} \lambda_{T} \kappa d y}{\int_{0}^{1} \lambda_{T} \kappa d y}\right] \frac{\partial f_{w}}{\partial y}=0
$$

This equation represents the second important result of this paper. The equation is a first-order, hyperbolic PDE, but in two spatial dimensions $(x, y)$, and it contains only saturation $S$ as the dependent variable. When $\kappa$ and $S$ are independent of $y$, it naturally reduces to the traditional 1-D Buckley-Leverett (B-L) equation

$$
\phi \frac{\partial S}{\partial t}+\frac{\partial f_{w}}{\partial x}=0
$$

In this sense, equation (11) is a $2-\mathrm{D}$ generalization of the B-L equation under the conditions of VFE. We must point out, however, that because of the reduction in the dimensionality, equation (11) cannot satisfy all boundary conditions, thus it is not expected to hold near all boundaries. This becomes more apparent below.

Before we proceed, let us recall the conditions for the validity of the above. It was derived in the limit $R_{L} \gg 1$ in the absence of gravity and capillarity. The latter condition requires that the gravity terms in $(3 \mathrm{~d})$ are small, or 


$$
N_{G} \ll 1
$$

where the gravity number was defined, $N_{G} \equiv \frac{H k_{H} g\left(\rho_{w}-\rho_{0}\right)}{L \mu_{o} q}$ (note the difference in notation with Fayers and Muggeridge, 1990). This allows for both equations (8d) and (9) to remain valid. However: in order for gravity and capillarity to be also absent from (4) and (11), one needs the stronger constraints (see also below)

$$
N_{G} \ll \frac{1}{R_{L}^{2}} \text { and } N_{C T} \ll \epsilon
$$

where the transervse capillary number is $N_{C T} \equiv \frac{\sqrt{k_{V}}}{H N_{c a}}$ (see Yokoyama and Lake, 1981). As pointed out in the introduction, under these conditions, equation (11) is a VFE approximation that emphasizes viscous cross-flow and heterogeneity. It is assumed, therefore, to control viscous fingering in such systems.

\section{LAYERED RESERVOIRS}

While the fuil solution of (11) is possible, it is more practical to consider a discrete treatment of the $y$-dependence instead. For example, this would be the case of a layered reservoir (Fig. 4). We take

$$
\begin{aligned}
& \kappa(x, y) \simeq \kappa_{i}(x) \\
& S(x, y) \simeq S_{i}(x)
\end{aligned} ; \frac{i-1}{N}<y<\frac{i}{N} ; \quad i=1, N
$$

Here, $N$ is the number of equal thickness layers of the system. For simplicity, we shall denote $\psi=\lambda_{T} \kappa / \int_{0}^{1} \lambda_{T} \kappa d y$. We may then integrate (11) ove $y$ from $\frac{i-1}{N}$ to $\frac{i}{N}$, to obtain the system

$$
\phi_{i} \frac{\partial S_{i}}{\partial t}+\psi_{i} \frac{\partial f_{i}}{\partial x}-\left(f_{i}-f_{i-1}\right) \frac{\xi}{\partial x}-\sum_{1}^{i-1} \psi_{j}=0 ; i=1, N
$$

Here, we have approximated $y$-integrals hy sums, have denoted $f_{i} \equiv f_{w}\left(S_{i}\right), \psi_{i} \equiv \psi\left(S_{i} ; \kappa_{i}\right)$ and defined $f_{o} \equiv \psi_{o} \equiv 0$. We may recast (16) in terms of a hyperbolic system

$$
\frac{\partial S}{\partial t}+\mathbf{A} \frac{\partial S}{\partial x}=\mathbf{B} \frac{\partial \kappa}{\partial x}
$$

where $\mathbf{S}=\left[S_{1}, S_{2}, \ldots, S_{N}\right]^{T}$ and $\kappa=\left[\kappa_{1}, \kappa_{2}, \ldots, \kappa_{N}\right]^{T}$ are $(N \times 1)$ vectors. $\mathbf{A}=\left[a_{i j}\right]$ and $\mathbf{B}=\left[b_{i j}\right]$ $(i, j=1, N)$ are $N \times N$ square matrices with the following coefficients. Matrix $\mathbf{A}$ consists of two 
terms $\mathbf{A}=\mathbf{D}-\mathbf{E}$, where

$$
\begin{aligned}
d_{i i} & =\frac{\lambda_{T}\left(S_{i}\right) \kappa_{i}}{\phi_{i} \Lambda}\left(\frac{\partial f_{w}}{\partial S}\right)_{i} \\
d_{i j} & =0, i \neq j
\end{aligned}
$$

and

$$
\begin{aligned}
e_{i j}= & \frac{\left(f_{w}\left(S_{i}\right)-f_{w}\left(S_{i-1}\right)\right.}{\phi_{i} \Lambda^{2}} \kappa_{j} \times \\
& \left(\frac{\partial \lambda_{T}}{\partial S^{\prime}}\right)_{j} \begin{cases}\frac{1}{N} \sum_{i}^{N} \lambda_{T}\left(S_{m}\right) \kappa_{m} & ; j \leq i-1 \\
-\frac{1}{N} \sum_{1}^{i-1} \lambda_{T}\left(S_{m}\right) \kappa_{m} & ; j \geq i\end{cases} \\
& e_{i i}=0
\end{aligned}
$$

The inhomogeneous matrix $\mathbf{B}$ has coefficients

$$
\begin{aligned}
& b_{i j}= \frac{\left(f_{w}\left(S_{i}\right)-f_{w}\left(S_{i-1}\right)\right)}{\phi_{i} \Lambda^{2}} \times \\
& \lambda_{T}\left(S_{j}\right) \begin{cases}\frac{1}{N} \sum_{i}^{N} \lambda_{T}\left(S_{m}\right) \kappa_{m} & ; j \leq i-1 \\
-\frac{1}{N} \sum_{1}^{i-1} \lambda_{T}\left(S_{m}, \kappa_{m}\right. & ; j \geq i\end{cases}
\end{aligned}
$$

In the above we have denoted $\Lambda \equiv \frac{1}{N} \sum_{i}^{N} \lambda_{T}\left(S_{m}\right) \kappa_{m}$.

This general formulation in discrete form is free of empirical arguments and represents a rigorous result, apparently also obtained here for the first time.

We observe the following:

1. Matrix $\mathbf{A}$ is neither diagonal nor symmetric. This is because of the coupling between adjacent layers of different properties. The coupling is due to the variation of the mobility with saturation.

2. When the layer permeabilities also depend on position $\left(\frac{\partial \kappa}{\partial x} \neq 0\right)$ a source ( $\left.\operatorname{sink}\right)$ term arises on the RHS of (17). Thus, in this case, heterogeneity acts in the form of a reaction term. 
This feature is also duc to the coupling between the layers and it is absent in the single layer case.

3. When all layer properties are the same, the systern reciuces to the B-L equation, as expected.

4. For a two-layer system, we may further simplify $\left(\Lambda=\lambda_{T}\left(S_{1}\right) \kappa_{1}+\lambda_{T}\left(S_{2}\right) \kappa_{2}\right)$ to obtain

$$
\begin{gathered}
d_{11}=\frac{\lambda_{T}\left(S_{1}\right) \kappa_{1}}{\phi_{1} \Lambda}\left(\frac{\partial f_{w}}{\partial S}\right)_{1} \\
d_{22}=\frac{\lambda_{T}\left(S_{2}\right) \kappa_{-}}{\phi_{2} \Lambda}\left(\frac{\partial f_{w}}{\partial S}\right)_{2} \\
d_{12}=d_{21}=0 \\
e_{21}=\frac{1}{2}\left(\frac{f_{w}\left(S_{2}\right)-f_{w}\left(S_{1}\right)}{\phi_{2} \Lambda^{2}}\right) \kappa_{1} \kappa_{2}\left(\frac{\partial \lambda_{T}}{\partial S^{2}}\right)_{1} \lambda_{T}\left(S_{2}\right) \\
e_{22}=-\frac{1}{2}\left(\frac{f_{w}\left(S_{2}\right)-f_{w}\left(S_{1}\right)}{\phi_{2} \Lambda^{2}}\right) \kappa_{1} \kappa_{2}\left(\frac{\partial \lambda_{T}}{\partial S}\right)_{2} \lambda_{T}\left(S_{1}\right) \\
b_{22}=-\frac{1}{2}\left(\frac{f_{1 *}\left(S_{2}\right)-f_{u \prime}\left(S_{1}\right)}{\phi_{2} \Lambda^{2}}\right) \lambda_{T}\left(S_{1}\right) \lambda_{T}\left(S_{2}\right) \kappa_{1} \\
b_{21}=\frac{1}{2}\left(\frac{f_{w}\left(S_{2}\right)-f_{w}\left(S_{1}\right)}{\phi_{2} \Lambda^{2}}\right) \lambda_{T}\left(S_{1}\right) \lambda_{T}\left(S_{2}\right) \kappa_{2} \\
b_{12}=0
\end{gathered}
$$

The above contains the formulation of Zapata and Lake (1981) and Pande and Orr (1959), but it is here also augmented by permeability heterogeneity along the $x$-direction. The latter result is also new. 


\section{EFFECTS OF CAPILLARITY AND GRAVITY}

When the injection rates are low enough for capillary and gravity effects to be of some importance, but not very low for the phases to be segregated, equations (4) must be reformulated. We obtain

$$
\begin{array}{r}
u_{w}=u f_{w}(S)+\frac{N_{C T}}{R_{L}} \kappa_{H} \lambda_{o} f_{w} \frac{\partial \Pi_{c}}{\partial x} \\
v_{w}=v f_{w}(S)+\kappa_{V} \lambda_{o} f_{w}\left\{N_{C T} \frac{\partial \Pi_{c}}{\partial y}-\frac{\delta}{\epsilon} N_{G}\right\}
\end{array}
$$

where a $J$-function representation was used for the capillary pressure, and $\Pi_{c}$ is the dimensionless capillary pressure.

Equations (5), (11) are appropriately modified. For instance, (5a) becomes

$$
\begin{aligned}
& \epsilon\left[\phi \frac{\partial S}{\partial t}+\frac{\partial}{\partial x}\left(u f_{w}+\frac{N_{C T}}{R_{L}} \kappa_{H} \lambda_{o} f_{w} \frac{\partial \Pi_{c}}{\partial x}\right)\right]+ \\
& \frac{\partial}{\partial y}\left[v f_{w}+\kappa_{V} \lambda_{o} f_{w}\left\{N_{C T} \frac{\partial \Pi_{c}}{\partial y}-\frac{\delta}{\epsilon} N_{G}\right\}\right]=0
\end{aligned}
$$

We may proceed in exactly the same way as before, by substituting $v=\epsilon w$, identifying the large parameter as $R_{L}^{2}=\frac{\delta}{\epsilon^{2}}$ and expanding appropriately. In order for the flow not to be segregated, condition (13) must still hold, $N_{G} \ll 1$. The longitudinal capillary term can be reglected, except near sharp fronts, much like in the classical Buckley-Leverett problem. However, transverse capillarity and gravity can be retained, if the following conditions are valid, $N_{C T} \sim O(\epsilon)$ and $N_{G} \sim O\left(\frac{1}{R_{L}^{2}}\right)$. Then, the following equation is obtained

$$
\begin{array}{r}
\phi \frac{\partial S}{\partial t}+\frac{\lambda_{T \kappa}}{\int_{0}^{1} \lambda_{T} \kappa d y} \frac{\partial f_{w}}{\partial x}-\frac{\partial}{\partial x}\left[\frac{\int_{0}^{y} \lambda_{T} \kappa d y}{\int_{0}^{1} \lambda_{T} \kappa d y}\right] \frac{\partial f_{w}}{\partial y}= \\
-\frac{\partial}{\partial y}\left[\kappa \lambda_{o} f_{w}\left\{\frac{N_{C T}}{\epsilon} \frac{\partial \Pi_{c}}{\partial y}-R_{L}^{2} N_{G}\right\}\right]
\end{array}
$$

The relative importance of gravity over capillarity depends on the dimensionless ratio

$$
\frac{\epsilon R_{L}^{2} N_{G}}{N_{C T}}=\frac{g \Delta \rho H \sqrt{k_{V}}}{\gamma}
$$

which is rat :-independent. For thin beds of low vertical permeability, capillarity dominates. Then, if the typical assumption is made about $\Pi_{c}$ as a single function of $S$, the $R H S$ above represents 
capillary spreading (one should keep in mind, however, that permeability heterogeneity is likely to also imply capillary heterogeneity as well, see Yortsos and Chang, (1990). This case is of interest but will not be considered in this study). As shown below in the case of miscible displacement, capillary spreading can be equivalently represented in terms of a macro-dispersivity. Capillary effects in VFE were considered by Yokoyama and Lake (1981).

When gravity dominates over capillarity in (32), then

$$
\begin{array}{r}
\phi \frac{\partial S}{\partial t}+\frac{\lambda_{T} \kappa}{\int_{0}^{1} \lambda_{T} \kappa d y} \cdot \frac{\partial f_{w}}{\partial x}-\frac{\partial}{\partial x}\left[\frac{\int_{0}^{y} \lambda_{T} \kappa d y}{\int_{0}^{1} \lambda_{T} \kappa d y}\right] \frac{\partial f_{w}}{\partial y}= \\
+\Theta_{G} \frac{\partial}{\partial y}\left[\kappa V \lambda_{o} f_{w}\right]
\end{array}
$$

wliere we defined $\Theta_{G}=\frac{k_{Y} g}{\mu_{o} g}\left(\rho_{w}-\rho_{o}\right)$. Upon integration over $y$ between $\frac{i-1}{N}$ and $\frac{i}{N}$ the contribution of the gravity term acts as a source/sink. Then, equation (17) must be modified as follows

$$
\frac{\partial \mathbf{S}}{\partial t}+\mathrm{A} \frac{\partial \mathrm{S}}{\partial x}=\mathrm{B} \frac{\partial \kappa}{\partial x}+\mathbf{G}
$$

where the $i^{\text {th }}$ element of the $\mathbf{G}$ vector is

$$
g_{i}=N \Theta_{G}\left[\kappa_{V} \lambda_{o}\left(S_{i}^{\prime}\right) f_{w}\left(S_{i}\right)-\kappa_{V} \lambda_{o}\left(S_{i-1}\right) f_{w}\left(S_{i-1}\right)\right]
$$

If the flow rates are quite low, such that $N_{G} \sim O(1)$, the phases are likely to segregated. This case is described in a later section.

\section{MISCIBLE DISPLACEMENT}

Consider, next, a first-contact miscible process in the limit of negligible gravity, $N_{G} \ll 1$. The mathematical description consists of equations $(5 \mathrm{~b})-(5 \mathrm{~d})$, where the total mobility is now a normalized inverse viscosity, $\lambda_{T}(C)=\frac{1}{\mu(C)}$, and where the dimensionless concentration $C$ is the dependent variable. The latter satisfies an advection-dispersion equation, which reads in dimensional notation

$$
\begin{aligned}
& \epsilon\left(\phi \frac{\partial C}{\partial t}+u \frac{\partial C}{\partial x}\right)+v \frac{\partial C}{\partial y}= \\
& \frac{\epsilon}{P e_{L}} \cdot \frac{\partial^{2} C}{\partial x^{2}}+\frac{a_{T}}{\alpha_{L}} \frac{1}{\epsilon \epsilon_{L}} \frac{\partial^{2} C}{\partial y^{2}}
\end{aligned}
$$


This equation is the analogue of (5a) to miscible displacement. In the above, we have denoted with $\alpha_{L}$ and $\alpha_{T}$ the longitudinal and transverse dispersivities, respectively, and we expressed longitudinal dispersion with the Peclet number, $P e_{L}$. In the case of mechanical dispersion only,

$$
P e_{L} \simeq \frac{L}{\alpha_{L}}
$$

Typically, $L \gg \alpha_{L}$, thus longitudinal dispersion can be neglected (except near sharp fronts). To obtain asymptotic results we follow the same procedure as above to get the final result

$$
\phi \frac{\partial C}{\partial t}+\frac{\lambda \kappa}{\int_{0}^{1} \lambda \kappa d y} \frac{\partial C}{\partial x}-\frac{\partial}{\partial x}\left[\frac{\int_{0}^{y} \lambda \kappa d y}{\int_{0}^{1} \lambda \kappa d y}\right] \frac{\partial C}{\partial y}=N_{T D} \frac{\partial^{2} C}{\partial y^{2}}
$$

where, subscript $T$ was omitted for simplicity. In (39) we have neglected longitudinal dispersion, but have retained transverse dispervion by assuming that $N_{T D} \equiv \frac{\alpha_{T}}{\alpha_{L}} \frac{1}{\epsilon^{2} P e_{L}} \simeq \frac{\alpha_{T}}{\epsilon H}$ remains finite in the limit of small $\epsilon$. When transverse dispersion is neglected, the previous equation (11) is recovered, if the identification is made $S \leftrightarrow C, \lambda_{T} \leftrightarrow \lambda$, and $f_{w} \leftrightarrow C$.

In the latter case, the equivalent of (17) reads

$$
\frac{\partial \mathbf{C}}{\partial t}+\mathbf{A} \frac{\partial \mathbf{C}}{\partial x}=\mathbf{B} \frac{\partial \kappa}{\partial x}
$$

where $\mathbf{C}=\left[C_{1}, C_{2}, \cdots, C_{N}\right]^{T}$. The representation becomes simpler if we take the approximate mobility dependence $\lambda(C)=e^{r C}$, where $r$ measures the mobility ratio, $r=\ell n M$. Then, for constant $\phi_{i}=\phi$, which can subsequently be absorbed in the dimensionless time, the coefficient matrices reduce to the following

$$
\begin{aligned}
d_{i i} & \equiv d_{i}=\frac{\kappa_{i} e^{r C_{i}}}{\frac{1}{N} \sum_{1}^{N} \kappa_{j} e^{r C_{j}}}, \\
\epsilon_{i j} & =r\left(C_{i}-C_{i-1}\right) d_{j}\left\{\begin{array}{l}
\frac{1}{N} \sum_{i}^{N} d_{m}, j \leq i-1 \\
-\frac{1}{N} \sum_{1}^{i-1} d_{m}, i \leq j
\end{array}\right.
\end{aligned}
$$

and

$$
\iota_{i, j}=\frac{e_{i j}}{r r_{i j}}
$$


One notes the interesting property that the sum of all the elements of each column of $E$ vanishes, $\sum_{j=1}^{N} e_{i j}=0$.

When the solute is not passive, an exact solution to the system is not available. Some interesting remarks can be made in the two limits where $r-+\infty$ (unstable displacement, $M \gg 1$ ) or when $r \rightarrow-\infty$ (stable displacement, $M \ll 1)$. In these two cases it can be shown readily that the matrix $\mathbf{C}$ becomes diagonal and, furthermore, that the only element with non-zero velocity is the layer with the highest $(r \rightarrow \infty)$ or the lowest concentration $(r \rightarrow-\infty)$, respectively. To show this, consider (41a) and rearrange as follows

$$
d_{i}=\frac{\kappa_{i}}{\frac{1}{N} \sum_{1}^{N} \kappa_{j} e^{r\left(C_{j}-C_{i}\right)}}
$$

It is then straightforward that, in the limit $r \rightarrow \infty$, then $d_{i} \rightarrow 0$ for all $i$, except for $i=\max$, where $C_{\max }$ is the maximum concentration, hence $d_{\max } \rightarrow N$. The opposite applies for the case $r \rightarrow \infty$, in which $d_{i} \rightarrow 0$ for all $i$, except for $i=\min$, where $C_{\min }$ is the minimum concentration, hence $d_{\min } \rightarrow N$. In summary, in the case of very unstable displacement $(M \gg 1)$, the highest concentration travels the fastest, in accord with viscous fingering notions. While, for very stable displacement $(M \ll 1)$, it is the lowest concentration that travels the fastest, also as expected. Besides this simple result, however, equation (40) contains a much richer structure. This is currently under study.

\section{TRACER DISPERSION}

In the passive solute case, where the viscosity is constant $(r=0)$, the off-diagonal terms $e_{i j}$ vanish and we obtain the linear system

$$
\frac{\partial C_{i}}{\partial t}+r_{i} \frac{\partial C_{i}^{\prime}}{\partial x}=-\left(C_{i}-C_{i-1}\right) \frac{\partial}{\partial x}\left(\sum_{i}^{N} \kappa_{i m}\right)
$$

where, in the above $r_{i}$ is to be interpreted as normalized with the average permeability of a cross section. When $\frac{\partial \kappa_{2}}{\partial x}=0$, this has the solution

$$
r_{i}=I\left(t r_{i}-x\right)
$$


where $H(z)$ is the step function. In the continuum limit

$$
\bar{C}=\lim _{n \rightarrow \infty} \frac{1}{N} \sum_{1}^{N} C_{i}
$$

we further get

$$
\widetilde{C}=\int_{\kappa, \min }^{\kappa_{\max }} f(\kappa) H(\kappa t-x) d \kappa
$$

where $f(\kappa)$ is the $p d f$ of $\kappa$. If we define the cumulative distribution $F(\kappa)=\int_{\kappa_{\min }}^{\kappa} f(\kappa) d \kappa$, then we may rearrange to get

$$
\bar{C}=H\left(t \kappa_{\max }-x\right)-F\left(\frac{x}{t}\right)
$$

For a more general result in the case $\frac{\partial \kappa_{i}}{\partial x}=0$, we consider transverse dispersion. We shall make use of the continuum formulation (38) and write (for $\phi=$ const.)

$$
\frac{\partial C}{\partial t}+\kappa(y) \frac{\partial C}{\partial x}=N_{T D} \frac{\partial^{2} C}{\partial y^{2}}
$$

This equation represents Taylor-Aris dispersion as applied to porous media flows (Lake and Hirasaki, 1981). One can readily show that it tends asymptotically to macro-dispersion. Indeed, by using a coordinate moving with the average speed $\xi=x-t$, one gets (Tayior, 1953)

$$
(\kappa(y)-1) \frac{\partial C}{\partial \xi} \cong N_{T D} \frac{\partial^{2} C}{\partial y^{2}}
$$

which can be integrated to

$$
C=\frac{1}{N_{T D}} \frac{\partial \bar{C}}{\partial \xi} \int_{0}^{y} \int_{0}^{y^{\prime}}\left(\kappa\left(y^{\prime \prime}\right)-1\right) d y^{\prime \prime} d y^{\prime}
$$

In the moving frame of reference the mass flux over a cross-section is

$$
\begin{array}{r}
\int_{0}^{1}(\kappa(y)-1) C d y= \\
\frac{1}{N_{T D}} \int_{0}^{1}(\kappa(y)-1) \int_{0}^{y} \int_{0}^{y^{\prime}}\left(\kappa\left(y^{\prime \prime}\right)-1\right) d y^{\prime \prime} d y^{\prime} d y \cdot \frac{\partial \bar{C}}{\partial \xi}
\end{array}
$$

thus, yielding whe macro-dispersion approximation

$$
\frac{\partial \bar{C}}{\partial t}+\frac{\partial \bar{C}}{\partial x}=D_{m} \frac{\partial^{2} \bar{C}}{\partial x^{2}}
$$

where

$$
D_{m n} \equiv \frac{1}{N_{T D}} \int_{0}^{1}(\kappa(y)-1) \int_{0}^{y} \int_{0}^{y^{\prime}}\left(\kappa\left(y^{\prime \prime}\right)-1\right) d y^{\prime \prime} d y^{\prime} d y
$$


In dimensional notation, the macrodispersity is expressed as

$$
\begin{gathered}
\alpha_{m}=\frac{1}{H \alpha_{T}\left(k_{H}\right)^{2}} \int_{0}^{H}\left(K_{H}(y)-k_{H}\right) \\
\int_{0}^{y} \int_{0}^{y^{\prime}}\left(K_{H}\left(y^{\prime \prime}\right)-k_{H}\right) d y^{\prime \prime} d y^{\prime} d y
\end{gathered}
$$

This result was first derived by Lake and IIrasaki (1981) by different means.

\section{GRAVITY SEGREGATED FLOW}

Consider, next, the case where the gravity terms in (3) are strong, $N_{G} \sim O(1)$. Here, the approach is somewhat different. Gravity effects must be also considered in the distribution of pressure, not only in the equation for the mass balance. Consider, first, negligible capillarity in the immiscible displacement case. The general expression for the vertical flow velocity was derived before

$$
\frac{\epsilon}{\delta} v=-\kappa_{v}\left[\lambda_{T} \frac{\partial p}{\partial y}+N_{G}\left(\lambda_{w} \bar{\rho}_{w}+\lambda_{o} \bar{\rho}_{o}\right)\right]
$$

where $\bar{\rho}_{i} \equiv \frac{\rho_{i}}{\rho_{w}-\rho_{o}}$. In view of the fact that $v=\epsilon w$, we must take

$$
\lambda_{T} \frac{\partial p}{\partial y}+N_{G}\left(\lambda_{w} \bar{\rho}_{w}+\lambda_{o} \bar{\rho}_{o}\right)=0
$$

thus gravity terms must be considered in the distribution of pressure. This is a true VFE. However, the fluids now become segregated and the previous analysis is not necessary. To show that flow segregation occurs we consider (3ob) in the absence of capillary effects

$$
v_{w}=\epsilon\left[w f_{w}(S)-\kappa_{V} \lambda_{o} f_{w} N_{G} R_{L}^{2}\right]
$$

All terms above must be $O(1)$ or less. Ilowever, since we assumed $N_{G} \sim O(1)$, the last term on the RHS would diverge at large $R_{L}$, unless $k_{V} \lambda_{o} f_{w}$ vanishes, thus

$$
\lambda_{\dot{v}} f_{w} \rightarrow 0
$$

The solution of equation (59) is segregated flow

$$
S=\left\{\begin{aligned}
S_{u r r} & ; \quad h<y<1 \\
1-S_{u r} & ; \quad 0<y<h
\end{aligned}\right.
$$


where the location of the "macroscopic interface" $h \equiv h(x, t)$ is to be determined as a function of position and time (Figure 2). This is the classical case of gravity tonguing which as shown above, holds under the conditions $R_{L} \gg 1, N_{G} \sim O(1)$. The first is a geometric condition, while the second also involves flow rates. To our knowledge, this is the first time that flow segregation was proved analytically.

To obtain the evolution of $h$, one needs to consider an integral balance. For this, equation (6a) is integrated over $y$ between 0 and 1 to yield

$$
\phi \frac{\partial}{\partial t} \int_{0}^{1} S d y+\int_{0}^{1} \frac{\partial}{\partial x}\left(u f_{w}\right) d y=0
$$

Next, we use (57) to solve with respect to $p$. We obtain after considerable algebra

$$
\frac{\partial p}{\partial x}=N_{G} \frac{\partial}{\partial x}\left[\int_{1}^{y} \frac{\left(\lambda_{w} \bar{\rho}_{w}+\lambda_{o} \bar{\rho}_{o}\right) d y}{\lambda_{T}}\right]+\frac{\partial \Pi}{\partial x}
$$

where $\Pi \equiv \Pi(x, t)$ is the presure at $y=1$ and depends on $x$ and $t$ only. Our ultimate goal is to obtain an expression for $u$. By subsequent substitution of (62) into (3b) and (61), we finally get

$$
\begin{array}{r}
\phi \frac{\partial}{\partial t} \int_{0}^{1} S d y+N_{G} \int_{0}^{1} \frac{\partial}{\partial x} \\
\left\{\kappa_{H} \lambda_{T} f_{w} \frac{\partial}{\partial x}\left(\int_{1}^{y}\left(\frac{\lambda_{w} \bar{\rho}_{w}+\lambda_{o} \bar{\rho}_{o}}{\lambda_{T}}\right) d y^{\prime}-\Pi\right)\right\} d y=0
\end{array}
$$

Next, the total mass balance is considered. In a straightforward manner it can be shown that the following equation results

$$
\begin{array}{r}
N_{G} \int_{0}^{1} \frac{\partial}{\partial x} \\
\left\{\kappa_{H} \lambda_{T} \frac{\partial}{\partial x}\left(\int_{1}^{y}\left(\frac{\lambda_{w} \bar{\rho}_{w}+\lambda_{o} \bar{\rho}_{o}}{\lambda_{T}}\right) d y^{\prime}-\Pi\right)\right\} d y=0
\end{array}
$$

The two equations must be solved in conjection with the distribution (60). We illustrate this application below.

\section{THE DUPUIT AND DIETZ APPROXIMATIONS}

Under the full segregation assumption, consider that the "oil" phase is "air" (switch for a moment. to subscript $a$ ) so that we take $\mu_{a} \ll \mu_{u}$ and $I I=$ const. This conveniently eliminates the last terms 
in (63) and (64). Expression (60) can be used to evaluate all the integrals. For example, we have

$$
\frac{\lambda_{w} \bar{\rho}_{w}+\lambda_{a} \bar{\rho}_{a}}{\lambda_{T}}= \begin{cases}\tilde{\rho}_{a} & ; \quad h<y<1 \\ \tilde{\rho}_{w} & ; \quad 0<y<h\end{cases}
$$

Hence,

$$
\int_{1}^{y}[] d y^{\prime}= \begin{cases}(y-1) \ddot{\rho}_{a} & ; \quad h<y<1 \\ (h-1) \bar{\rho}_{a}+(y-h) \bar{\rho}_{w} & ; \quad 0<y<h\end{cases}
$$

thus,

$$
\frac{\partial}{\partial x} \int_{1}^{y}[] d y^{\prime}= \begin{cases}0 & ; \quad h<y<1 \\ \left(\bar{\rho}_{a}-\bar{\rho}_{w}\right) \frac{\partial h}{\partial x} & ; \quad 0<y<h\end{cases}
$$

Proceeding similarly with the evaluation of the other integrals the final result is obtained

$$
\phi \frac{\partial h}{\partial t}=N_{G}^{\prime} \frac{\partial}{\partial x}\left[\int_{0}^{h} \kappa_{H}(x, y) d y \cdot \frac{\partial h}{\partial x}\right]
$$

where $N_{G}^{\prime}=N_{G} / M$ and $M$ is the ratio of the mobilities of the displacing to the displaced phase, (here $\left.M=\frac{\mu_{w} k_{r a}\left(S_{w r}\right)}{\mu_{a} k_{r w}\left(1-S_{a r}\right)}\right)$. This is the standald, non-linear diffusion equation used traditionally in the water infiltration literature (Fig. 5). It usually arises under the so-called Dupuit approximation where $\kappa_{H}=1$ (see Bear, 1972). Here, it was derived explicity, rigorously, and more generally.

In the more general case, a similar approach applies. The evaluation of the various integrals is much simplified, if generalized functions (like step and delta functions and their derivatives) are used. For instance, we can take

$$
\lambda_{T}=\frac{1}{M} I(y-h)+I I(h-y)
$$

so that the integral in (63) is expressed in the compact form $\bar{\rho}_{o}(y-1) H(y-h)+\left(\bar{\rho}_{o}(h-1)+\right.$ $\left.\bar{\rho}_{w}(y-h)\right) H(h-y)$, etc. The properties of the generalized functions needed are $I^{\prime}(z)=\delta(z)$ and $z \delta^{\prime}(z)=-\delta(z)$, where $\delta(z)$ is the delta function of $z$. Without going into the considerable details, we shall only present the final results. The total mass balance yields

$$
\begin{aligned}
& N_{\zeta} \frac{\partial}{\partial x}\left[\int_{0}^{h} r_{H} d y \cdot \frac{\partial h}{\partial x}\right]- \\
& \frac{\partial}{\partial x}\left[\left(\int_{0}^{h} r_{H} d y+\frac{1}{J} \int_{h}^{1} i_{H} d y\right) \cdot \frac{\partial I I}{\partial x}\right]=0
\end{aligned}
$$


while the "water" mass balance becomes

$$
\begin{aligned}
& \phi \frac{\partial h}{\partial t}+M N_{G} \frac{\partial}{\partial x}\left[\int_{J_{0}}^{h} \kappa_{H} d y \cdot \frac{\partial h}{\partial x}\right]- \\
& M \frac{\partial}{\partial x}\left[\int_{0}^{h} \kappa_{H} d y \cdot \frac{\partial \Pi}{\partial x}\right]=0
\end{aligned}
$$

Here, $M=\frac{\mu_{o} k_{r w}\left(1-S_{o r}\right)}{\mu_{w} k_{r o}\left(S_{w r}\right)}$. We can integrate (70) once with respect to $x$ to get

$$
\begin{aligned}
& N_{G} \int_{0}^{h} \kappa_{H} d y \cdot \frac{\partial h}{\partial x}- \\
& \left(\int_{0}^{h} \kappa_{H} d y+\frac{1}{M} \int_{h}^{1} \kappa_{H} d y\right) \frac{\partial \Pi}{\partial x}=C
\end{aligned}
$$

where $C=1$, without loss, and then eliminate $\frac{\partial \Pi}{\partial x}$ between (71) and (72) to obtain the final equation

$$
\begin{array}{r}
\phi \frac{\partial h}{\partial t}+\frac{\partial}{\partial x}\left[\frac{\int_{0}^{h} \kappa_{H} d y}{\int_{0}^{h} \kappa_{H} d y+\frac{1}{M} \int_{h}^{1} \kappa_{H} d y}\right]= \\
N_{G} \frac{\partial}{\partial x}\left[\frac{\int_{0}^{h} \kappa_{H} d y \int_{h}^{1} \kappa_{H} d y}{\int_{0}^{h} \kappa_{H} d y+\frac{1}{M} \int_{h}^{1} \kappa_{H} d y} \frac{\partial h}{\partial x}\right]
\end{array}
$$

This is the generalization of the well known parallel flow approximation, derived by Dietz (1954) for the case of constant permeability. The process can be approximated as an 1 - D displacement with equivalent "saturation" $\bar{S}=S_{w r}+h\left(1-S_{o r}-S_{v r}\right)$, equivalent relative permeabilities given by $\bar{k}_{r w}=k_{r w}\left(1-S_{o r}\right) \int_{0}^{h} \kappa_{H} d y$ and $\bar{k}_{r o}=k_{r o}\left(S_{o r}\right) \int_{h}^{1} \kappa_{H} d y$, and a straight line "capillary pressure" with dispersion coefficient $N_{G}$.

The pseudofunctions become straight-lines when the permeability is constant (Dietz, 1953). In any other case, they are non-linear functions of $\bar{S}$, and, in fact, they may also vary with position $x$. Clearly, because of the integral form, the order of the layers affects drastically the shape of the pseudof'nctions (Lake et al, 1990).

\section{CAPILLARITY-GRAVITY SEGREGATION}

When the dimensionless ratio in (33) is not large, capillarity is also of importance. The equivalent of $(58)$ is now 


$$
v_{w}=\epsilon\left[w f_{w}\left(S^{\prime}\right)+t_{V} \lambda_{o} f_{w}\left(\frac{N_{C T}}{\epsilon} \frac{\partial \Pi_{C}}{\partial y}-N_{G} R_{L}^{2}\right)\right]
$$

Following the same arguments as before, when $N_{C T}$ and $N_{G}$ are not small, we are led to the capillary equilibrium condition

$$
N_{C T} \frac{\partial \Pi_{c}}{\partial y}=\frac{\delta}{\epsilon} N_{G}
$$

which can be integrated to yield

$$
\Pi_{c}=\Pi_{c o}+y \frac{\delta N_{G}}{\epsilon N_{C T}}
$$

where $\Pi_{c o}$ is the capillary pressure at $y=0$. If $\Pi_{c}$ is assumed to be a single function of saturation, the above determines the vertical distribution of saturation, given its value $S_{0}(x, t)$ at $y=0$.

Again, an integral approach is needed. However, the problem here is quite simpler. Indeed, it can be readily shown that the "water" pressure is hydrostatic, such that

$$
p_{w}=-N_{G} \bar{\rho}_{w}(y-1)+\Pi(x)
$$

hence, the total flow rate $u$ is described by expression (9) (assuming negligible capillarity along the $\mathrm{x}$-direction). Thus, we can use directly equation (61) to get

$$
\phi \frac{\partial}{\partial t} \int_{0}^{1} S d y+\frac{\partial}{\partial x}\left\{\frac{\int_{0}^{1} \kappa_{H} \lambda_{w}(S) d y}{\int_{0}^{1} \kappa_{H} \lambda_{T}(S) d y}\right\}=0
$$

Equations (76) and (78) completely specify the problem. For example, all integrals in (78) can be explicitly calculated from the solution of $(76)$, in terms of $S_{0}(x, t)$, the evolution of which can be obtained from (78). The result would be an equivalent to the Buckley-Leverett equation, this time in terms of $S_{0}(x, t)$. Whether, however, appropriate pseudofunctions can be defined remains to be investigated.

Finally, if capillarity predominates in equation (76), the saturation profiles along the vertical direction follow the capillary heterogencity. Specifically, if $k_{V}$ does not vary greatly with $y$, then the saturation profile is flat, $S=S_{0}(x, t)$, and equation (78) becomes the traditional Buckley-Leverett equation (12). 


\section{SUMMARY}

Ir this paper, using a formal approach, the various manifestations of Vertical Flow Equilibrium were derived. Key to the analysis was the identification of the parameter $R_{L}$ as the proper asymptotic variable and the development of a formal asymptotic method in terms of $1 / R_{L}^{2}$. The analysis confirms previously known numerical results and, for the first time, it rigorously establishes their validity in the limit of large $R_{L}^{2}$. Because the condition is geometric-structural it applies independently of flow and process parameters, hence it can be used regardless of the particular displacement process. Due to the ensuing reduction in the dimensionality of the problem, the process description is facilitated significantly. To our knowledge, this is one of the few cases in multiphase flow in porous media where such a reduction is possible.

The formal approach presented has many advantages, as it allows for a plethora of special cases to be readily derived. An analysis along these lines is also possible for any EOR process, and we hope to report on this in the future. In all cases, heterogeneity is the key variable of the description and it is only the relative interplay of viscous to other forces that dictates the various approximations. Viscous, gravity and capillary effects were considered in the case of immiscible displacement. The classification of the various regimes depends on the wrelative importance of these forces, as described by the dimensionless parameters. A summary is given in Table 1. A similar table can be constructed for miscible displacement, where the role of capillarity is played by transverse dispersion. We should point out that the above can be readily extended to include a second "horizontal" dimension by appropriate, b. t perhaps laborious, modifications.

Many of the results obtained here are new, in the sense that they extend previous approximate analyses. Thus, eqns. (11) and (17) are an extension of Zapata and Lake (1981), eqns (32) and (34) extend the work of Yokoyama and Lake (1981), eqns (40) and (53) are extensions of Lake and Hirasaki (1981), and eqns (68), ( 73 ) and (78) extend the Dupuit and Dietz approximations. In addition to their formal aspects, our results also ofter insight on effects of viscous cross-flow, as in (18)-(20), and they suggest directly the relevant pscudofunctions for each case. Finally, it is the hope that a more detailed analysis of equation (40) would lead to improved approximations and to rigorously establishing the validity of the various cmpirical viscous fingering models (such as Kicval, 1963, Todd and Longstaff, 1979 and Faynrs, 1988). 


\section{ACKNOWLEDGEMENTS}

The research of the author is supported in part by DOE contract FG22-90BC14600, and by the Center for the Study of Fractured Reservoirs supported by Oryx, Shell E \& P, Texaco, Unocal, and the California State Lands Commission. This work originated from a discussion with Karin Pande. The author would like to thank Murat Zeyluek for his assistance.

\section{REFERENCES}

Bear, J., Dynamics of Fluids in Porous Media, American Elsevier Publishing Company, Inc., New ; York (1972).

Beckers, H.L., "The Deformation of an Interface Between Two Fluids in a Porous Medium", Appl. Science Res. Annual, 14, 101, (1965).

Coats, K.R., Dempsey, J.R. and Ilenderson, J.H., "The Usr, of Vertical Equilibrium in Two-Dimensional Simulation of Three-Dimensional Reservoir Performance", Soc. Petroleum Eng. J., 11, 63-71 (1971).

Dietz, D.N., "A Theoretical Approach to thn Problem of Encroaching and By-Passing Edge Water", Akad. van Wetenschappen. Amsterdam, Proc. 56-B. 83, (1954).

Fayers, F.J., "An Approximatate Model with Physically Interpretable Larameters for Representing Viscous Fingering", paper SPE $13166^{\circ}$ presented at the Society of Petroleum Engincers Fall Technical Conference and Exhibition, IJouston, Texas (1984).

Fayers, F.J., and Muggeridge, A.II., "Extensions to Dietz Theory and Behavior of Gravity Tongues in Slightly Tilted Reservoirs", SPE Reservoir Engineeri, o, 4\$7-494 (1990).

Loval. E.J.. "A Method for Predicting the Performanc of Unstable Miscible Displaceme.t in Ileterogencous Media”, Soc. Petroleum Eing. J., 3, 145-155, (1963).

Lake, L.W. and Ilirasaki. (i.J., "Taylor"s Dispersion in Suatified Porous Media", Soc. Pet. Eng. J., $21.459-40 \% \cdot(1951)$. 
Lake, L.W., Kasap, E., and Shook, M., "Pseudofunctions - The Key to Practical Use of Reservoir Description", North Sea Oil and Gas Reservoirs - II, The Norwegian Institute of Technology, Graham \& Trotman, 297-308 (1990).

Le Fur, B., and Sourieau, P., "Étude de 1 Écoulement Diphasique dans une Couche Inclinée et dans un Modéle Rectangulaire de Milieu Poreux", Rev. Inst. Fr. Petrol. 18, 325-343, (1963).

Pande, K. K., and Orr, F.M. Jr., "Interaction of Phase Behavior, Reservoir Heterogeneity, and Crossflow in $\mathrm{CO}_{2}$ Floods", paper SPE 19668 presented at the Society of Petroleum Engineers Fall Technical Conference and Exhibition, San Antonio, Texas (1989).

Taylor, G.I., "Dispersion of Soluble Matter in Solvent Flowing Slowly Through a Tube", Proc. Roy. Soc. A, 219, 186-203 (1953).

Todd, M.R., and Longstaff, W.J., "The Development, Testing, and Application of a Numerical Simulator for Predicting Miscible Flood Performance", JPT, 874-82 (1972).

Yortsos, Y.C., and Chang, J., "Capillary Effects in Steady-State Flow in Heterogeneous Cores", TiPM, 5, 399-420 (1990).

Yokoyama, Y., and Lake, L.W., "The Iffects of Capillary Pressure on Immiscible Displacements in Stratified Porous Media", paper SPE 10109, presented at the Society of Petroleum Engineers Fall Technical Conference and Exhibition, San Antonio, Texas (1981).

Zapata, V.J., and Lake, L.W., "A Theoretical Analysis of Viscous Crossflow", paper SPE 10111 presented at the Society of Petroleum Engineers Fall Technical Conference and Exhibition, San Antonio, Texas (1981). 

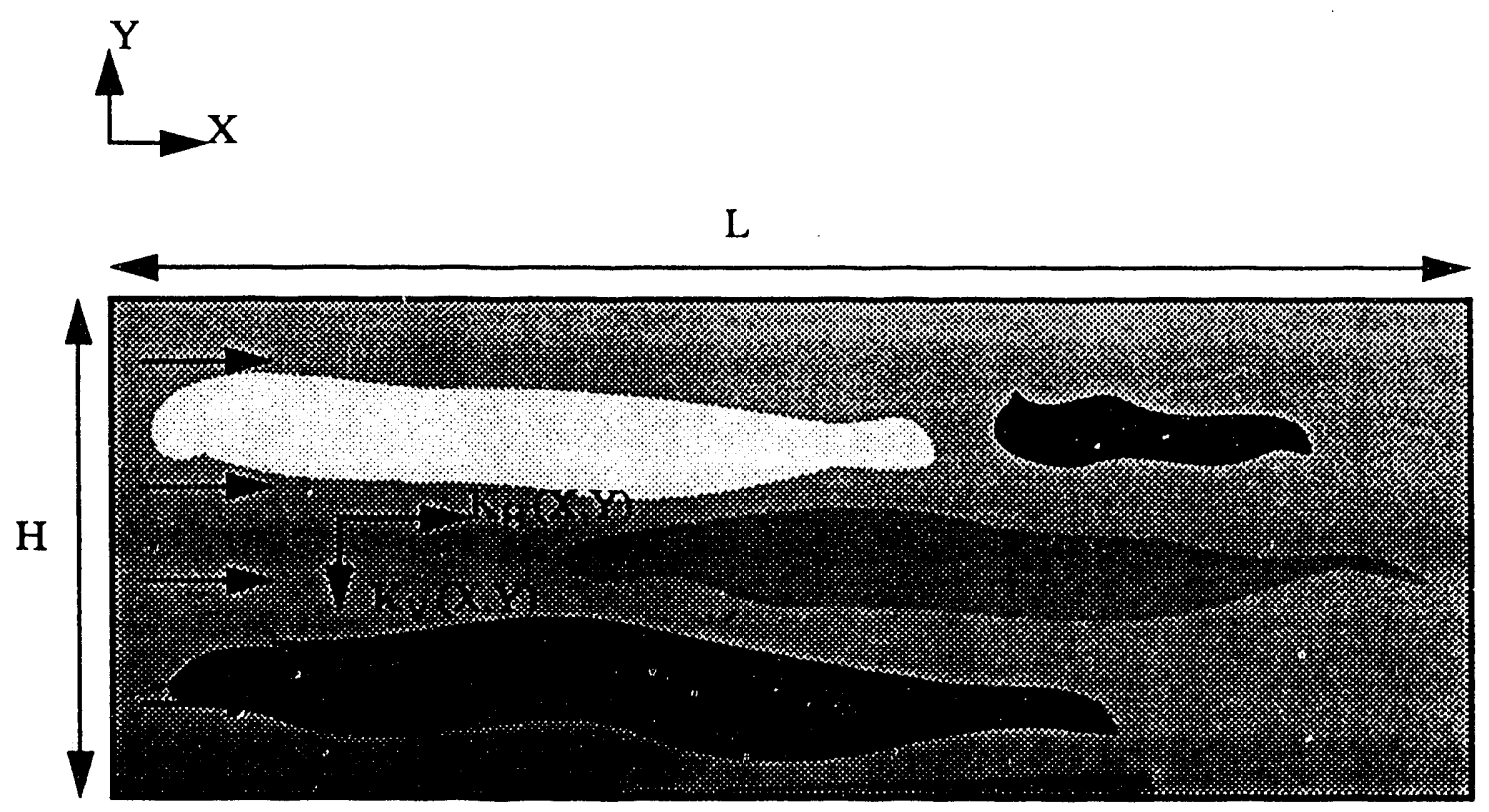

Figure 1: Schematic of Ileterogeneous Reservoir for VFE.
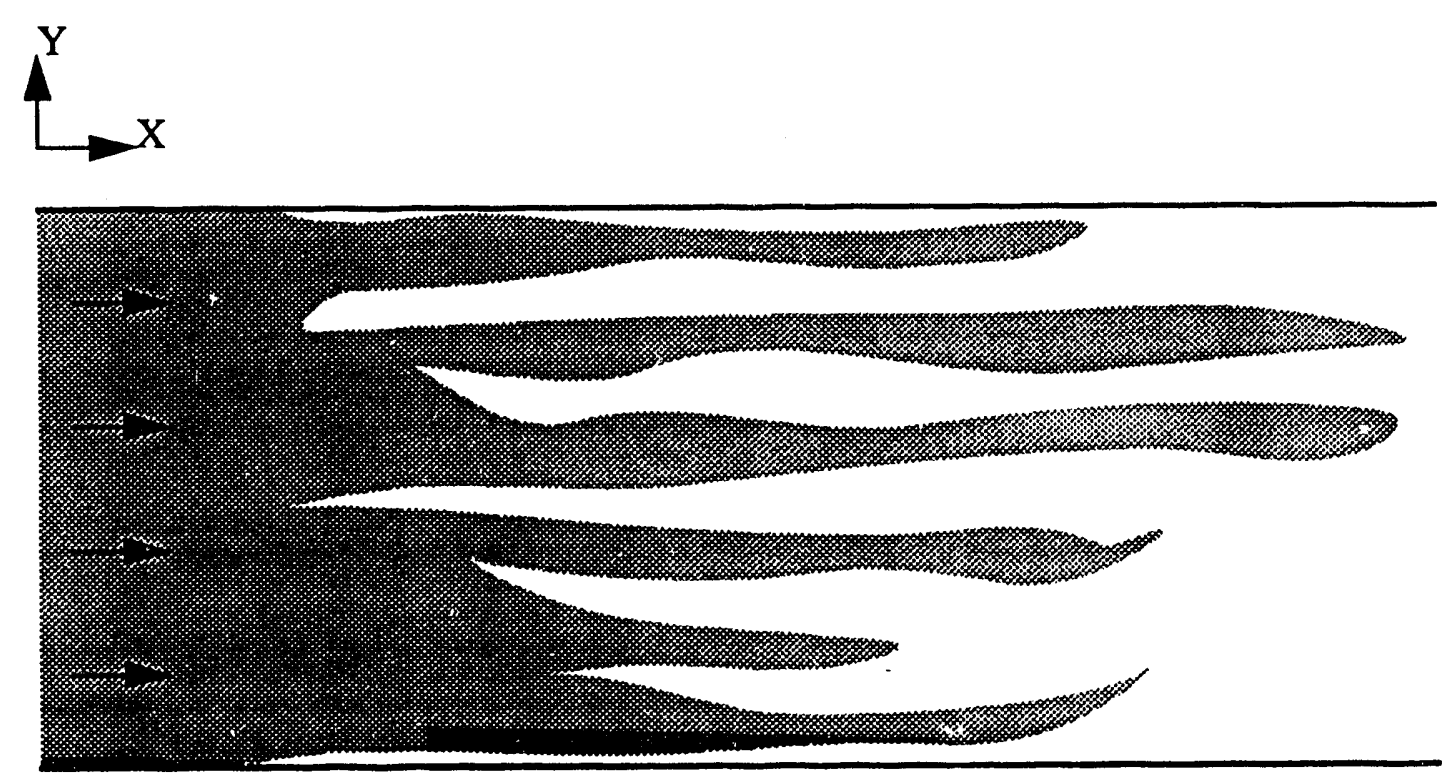

Figure 2: Schematic of Viscous Fingering in VFE. 


\begin{tabular}{|c|c|c|c|c|c|}
\hline Viscous & Gravity & Capillarity & Conditions & Equations & Comments \\
\hline Strong & Negligible & Negligible & $\mathrm{N}_{\mathrm{G}} \ll \frac{1}{\mathrm{R}_{\mathrm{L}}^{2}}, \mathrm{~N}_{\mathrm{CT}} \ll \varepsilon$ & $(11)$ and (17) & Viscous Fingering \\
\hline Strung & Moderate & Moderate & $\mathrm{N}_{\mathrm{G}} \sim \frac{1}{\mathrm{R}_{\mathrm{L}}^{2}}, \mathrm{~N}_{\mathrm{CT}}-\varepsilon$ & $(33)$ and (35) & $\begin{array}{c}\text { Viscous Fingering } \\
\text { with Dispersion }\end{array}$ \\
\hline Moderite & Strong & Negligible & $\mathrm{N}_{\mathrm{G}} \sim 1, \mathrm{~N}_{\mathrm{CT}} \ll \frac{\delta}{\varepsilon}$ & $(69$ and (74) & $\begin{array}{c}\text { Gravity Tonguing } \\
\text { (Dietz) }\end{array}$ \\
\hline Moderate & Strong & Moderate & $\mathrm{N}_{\mathrm{G}} \sim 1, \mathrm{~N}_{\mathrm{CT}}-\frac{\delta}{\varepsilon}$ & (79) & $\begin{array}{c}\text { Gravity-Capillary } \\
\text { Equilibrium }\end{array}$ \\
\hline Moderate & Moderate & Strong & $\mathrm{N}_{\mathrm{G}}-1, \mathrm{~N}_{\mathrm{CT}} \gg \frac{\delta}{\varepsilon}$ & (12) & $\begin{array}{c}\text { Capillary Equilibrium } \\
\text { (Buckley-Leverett) }\end{array}$ \\
\hline
\end{tabular}

Table 1: 

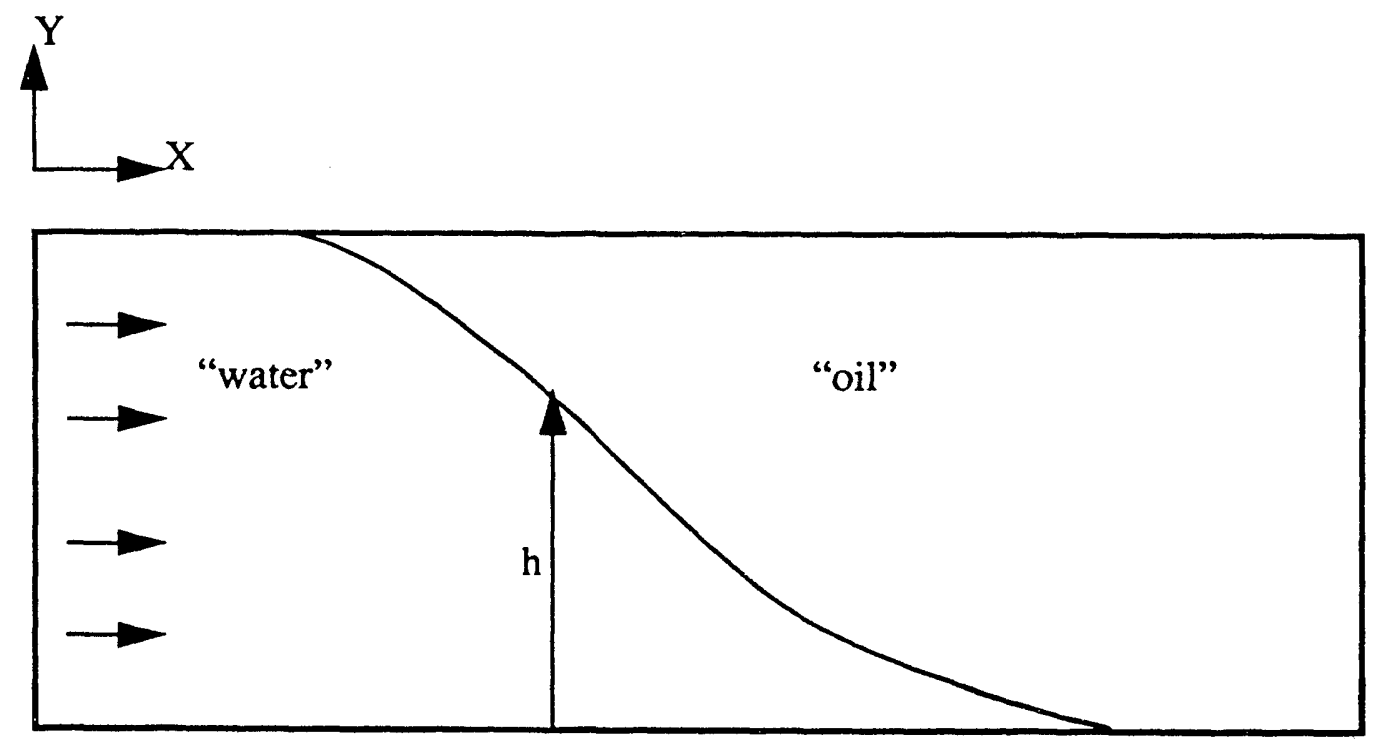

Figure 3: Schematic of Gravity Segregated Displacement.
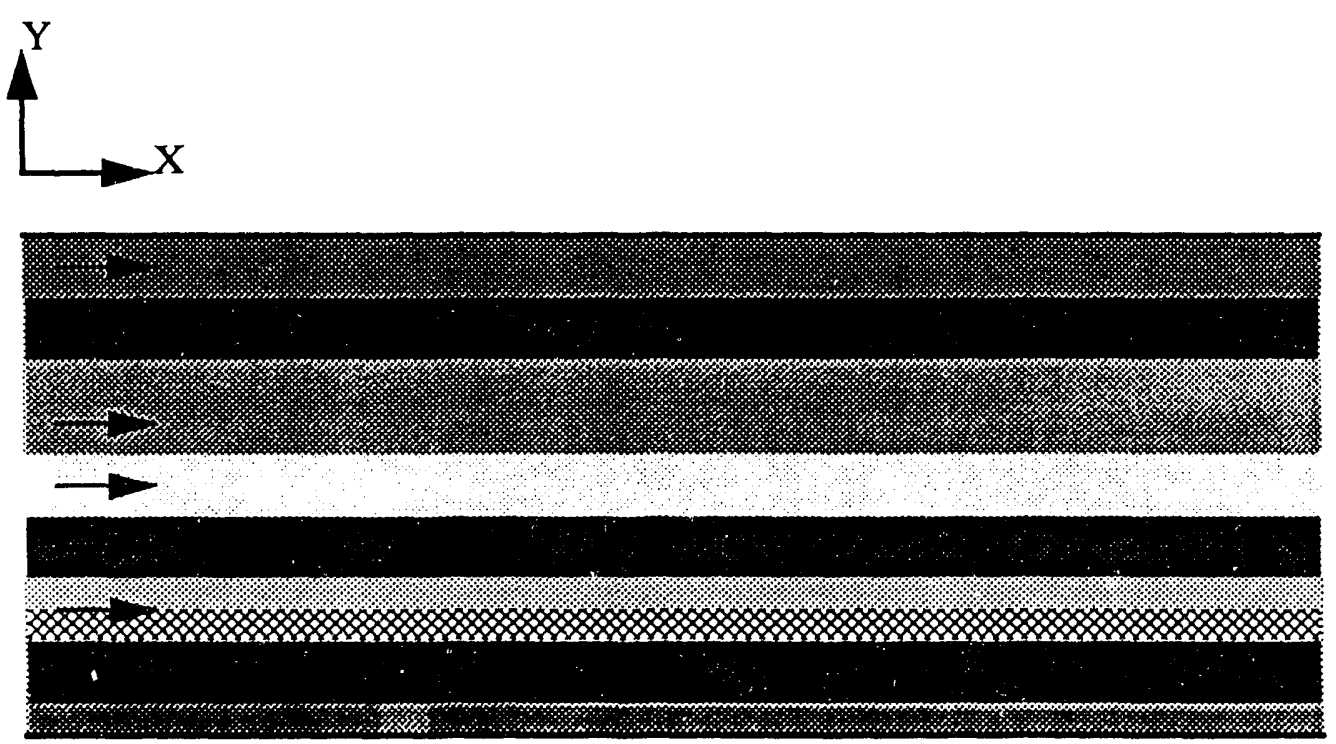

Figure t: Schematic of Layered Reservoir for VFE. 


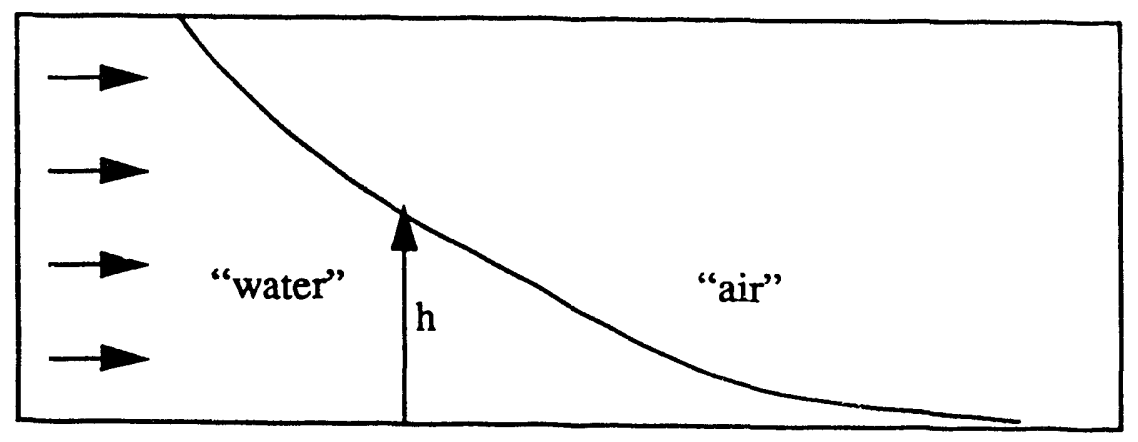

Figure 5: Schematic of Air-Water System for Dupuit Assumption. 

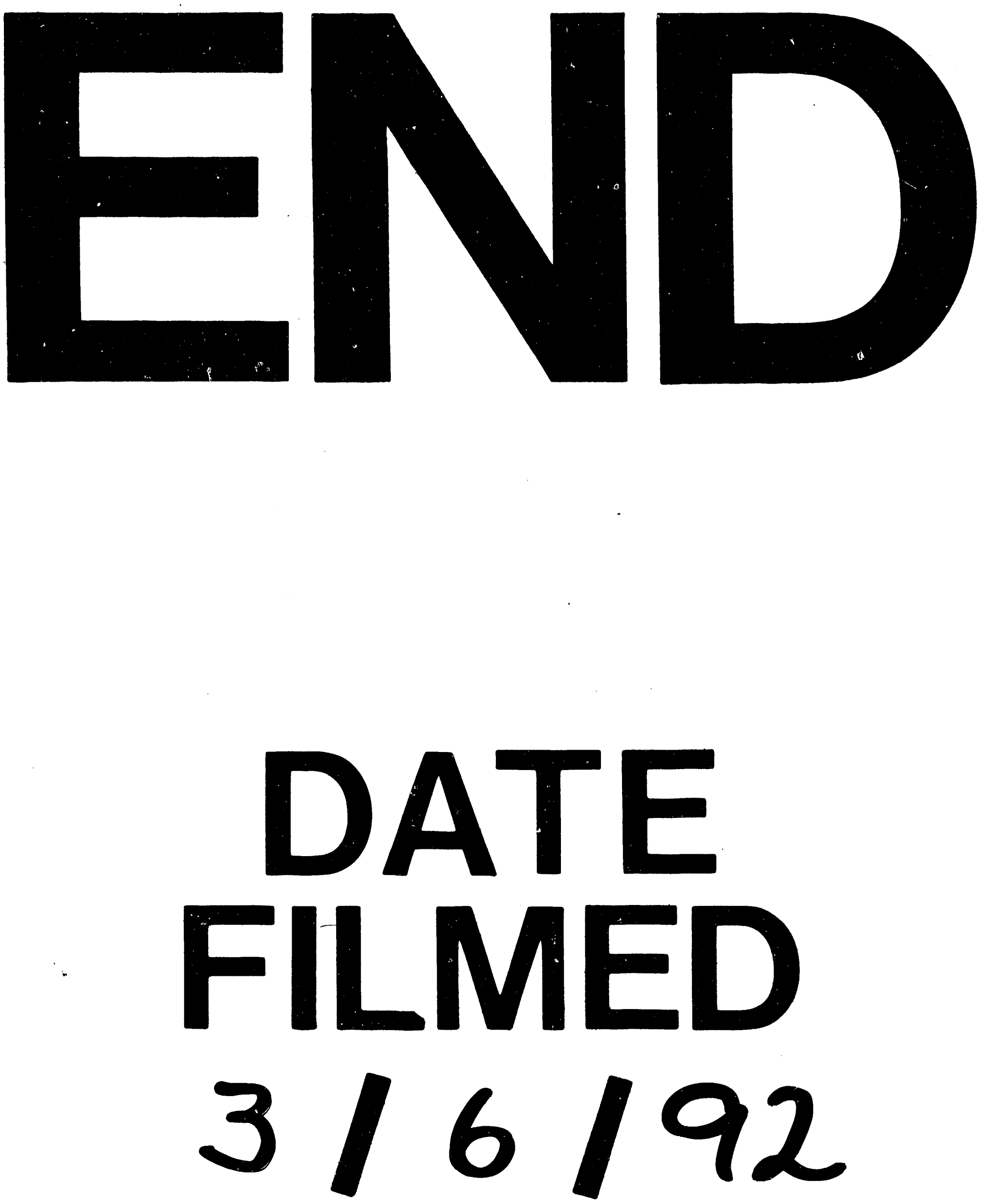

1 
